\title{
Sistema antioxidante, actividad física y consumo máximo de oxígeno en adultos jóvenes
}

\author{
Antioxidant system, physical activity and maximum oxygen consumption in young adults
}

\begin{abstract}
Liliana Aracely Enriquez-del-Castillo, Ramón Candia-Lujan1, Verónica Moreno-Brito², Lidia Guillermina De León Fierro', Sandra Alicia Reza-López ${ }^{2}$, Everardo González-Rodríguez², Claudia Esther Carrasco-Legleu ${ }^{1 *}$

Facultad de Ciencias de la Cultura Física, Universidad Autónoma de Chihuahua.

Facultad de Medicina y Ciencias Biomédicas. Universidad Autónoma de Chihuahua.
\end{abstract}

\section{RESUMEN}

La actividad física (AF) moderada y parámetros adecuados de composición corporal se sugiere que tienen efectos benéficos sobre el sistema antioxidante total (SAT). El consumo de oxígeno por ejercicio vigoroso genera producción de radicales libres. El objetivo de este trabajo fue analizar la relación del SAT, AF, consumo máximo de oxígeno ( $\mathrm{VO}_{2}$ máx) y composición corporal en adultos jóvenes. Se estudió una muestra no probabilística de 56 participantes con un promedio de edad de $20.7 \pm 2.1$ años. El nivel de AF se evaluó mediante cuestionario y el $\mathrm{VO}_{2}$ máx por la prueba Course-Navette; la composición corporal por impedancia bioeléctrica y el SAT por técnica colorimétrica en plasma. Los datos se analizaron mediante pruebas t-student o suma de rangos de Wilcoxon para determinar diferencias de acuerdo al género. Se analizó la relación entre las variables de interés mediante correlación de Pearson o Spearman. Los varones obtuvieron mayor $\mathrm{VO}_{2}$ máx que las mujeres $(\mathrm{p}=0.01)$. No se encontraron diferencias en SAT por género. Se encontró una correlación inversa entre SAT y METs reportados en varones $(r=-0.5 p=0.01)$. Se concluye que hay una modulación de SAT por el nivel de AF dependiente del género. No se encontró relación con el VO2máx y variables de composición corporal. Palabras Clave: Sistema Antioxidante Total, $\mathrm{VO}_{2}$ máx, nivel de actividad física, METs.

\section{ABSTRACT}

Evidence suggests that moderate physical activity (PA) and adequate parameters of body composition have beneficial effects on total antioxidant capacity (TAC). Oxygen consumption by vigorous exercise generates free radical production. The aim of this study was to analyze the relation of the TAC, PA, maximal oxygen uptake $\left(\mathrm{VO}_{2}\right.$ max) and body composition in young adults. A non-probabilistic sample of 56 participants were studied (mean age $=20.7 \pm 2.1$ years). PA level was evaluated by questionnaire and $\mathrm{VO}_{2}$ max by the Course-Navette test; body composition was analyzed by bioelectrical impedance and plasma TAC by colorimetric technique, and the data analyzed by t-student or Wilcoxon rank sum tests to determine differences according to gender. Pearson or Spearman correlation was used to analyze the relation between variables of interest. $\mathrm{VO}_{2}$ max values were greater in men than in women $(p=0.01)$. No differences were found in TAC by gender. TAC and METs values were inversely *Autor para correspondencia: Claudia Esther Carrasco-Legleu Correo electrónico: claudialegleu@hotmail.com correlated in men only $(r=-0.5, p=0.01)$. In conclusion, PA level modulates TAC in a gender dependent manner. $\mathrm{VO}_{2}$ max and body composition variables were unrelated.

Keywords: Total Antioxidant Capacity, $\mathrm{VO}_{2} \max$, physical activity level, METs.

\section{INTRODUCCIÓN}

A través de los años se han tratado de establecer parámetros que brinden información acerca de la salud de las personas (Ainswoorth, 2008) con la finalidad de mejorarla (Valdés, 2013), situación que ha llevado a un trabajo multidisciplinar en donde la actividad física (AF) es parte fundamental (Serra, 2008; Abarca-Sos et al., 2010). La AF genera un gasto energético, por lo que se requiere de una capacidad cardiorespiratoria (CCR) adecuada, para dar respuesta a las necesidades cardiovasculares que implica el ejercicio, principalmente a la demanda de una mayor cantidad de oxígeno (Valdés, 2014). Tanto la AF como la CCR pueden ser estimadas por métodos indirectos, ya que como menciona Hall-López et al. (2015), cuestionarios como el IPAQ permiten estimar el consumo semanal de METs (Equivalentes metabólicos) a través de medidas continuas registradas durante los últimos 7 días, o bien en medidas categóricas ya que dicho cuestionario permite clasificar al sujeto en un nivel de AF baja, moderada o vigorosa, además, Ochoa-Martinez et al. (2018) también utilizaron dicho cuestionario en adultos jóvenes universitarios. Mientras que para la evaluación de la CCR una opción confiable son las pruebas de campo como la Course Navette, la cual es utilizada para la determinación del consumo máximo de Oxígeno ( $\mathrm{VO}_{2}$ máx), misma que presenta una correlación directa con la intensidad de las actividades realizadas.

Con base en las recomendaciones acerca de la práctica de $A F$, realizarla de moderada intensidad al menos 150 minutos por semana para conservar la salud (Gutiérrez 2007; Garber et al., 2011) y tomando en cuenta el nivel de la misma actividad. Martinez-Vizcaíno y Sánchez-López en el 2008 mencionan que el realizar este tipo de AF de manera constante proporciona beneficios a la salud aumentando el sistema antioxidante (SAT) mediante la generación de antioxidante enzimáticos (Gutiérrez, 2007), y disminuyendo el riesgo de padecer enfermedades cardiovasculares. Sin embargo, el ejercicio de alta intensidad, supone la sobrecarga de radica- 
les libres (RL) y con ello daño celular y muscular (Peake et al., 2004; Könisberg, 2007), lo que genera un desequilibrio en el sistema oxidante/antioxidante llamado estrés oxidativo (EO), cuyo daño se extiende a diversas biomoléculas importantes como proteínas, lípidos y el DNA. Los RL están presentes en situaciones alterando el equilibrio del organismo, por lo que el cuerpo humano genera la necesidad de desarrollar métodos eficaces a través de la generación de antioxidantes endógenos (Torres et al., 2015). La principal función que tienen los antioxidantes es ayudar a reducir los efectos del EO a través de sustancias endógenas como enzimas y coenzimas, Velamazán en el 2005 sugirió que las personas que realizan algún tipo de AF aerobia por tiempo prolongado, logran aumentar de manera significativa sus reservas de antioxidantes en los tejidos respecto de quienes no lo hacen. En ese mismo año, Ozcelik y su grupo de investigación observaron que tras 12 semanas de entrenamiento aerobio en un grupo de pacientes con obesidad, se logró mejorar sus niveles plasmáticos de antioxidantes. De esta manera es posible estudiar la relación entre el nivel de $\mathrm{AF}_{1} \mathrm{VO}_{2}$ máx y el SAT, ya que la práctica de ejercicio de intensidad moderada y el entrenamiento formal pueden aumentar el $\mathrm{VO}_{2}$ máx de los sujetos y con ello observar niveles más altos de antioxidantes. (Aguiló et al., 2007; Torres et al., 2015; Sacheck et al., 2001).

Una manera indirecta por la cual puede ser evaluado el daño oxidativo es a través de la medición del SAT ya que evidencia cómo se encuentra de manera sistémica la respuesta antioxidante (Quintanar et al., 2009; Tobias et al., 2013). Con base en lo anterior el objetivo fue analizar la relación del SAT con la AF, el $\mathrm{VO}_{2}$ máx y componentes corporales en adultos jóvenes.

\section{MATERIALES Y MÉTODOS}

Se reclutó una muestra no probabilística de 56 adultos jóvenes entre 21 y 27 años de edad, de los cuales 39 fueron mujeres y 17 hombres. Se excluyeron los sujetos con algún padecimiento cardiaco, con obesidad mórbida, o mujeres en etapa gestacional. A quienes aceptaron participar se les solicitó la firma de una carta de consentimiento informado. Los procedimientos se realizaron bajo los principios establecidos en la declaración de Helsinki (Asociación Médica Mundial 2014).

Las variables antropométricas de peso, estatura, circunferencia de cintura y circunferencia de cadera fueron medidas por antropometristas certificados, bajo los lineamientos de la Sociedad Internacional para el Desarrollo de Cineantropometría (ISAK por sus siglas en inglés) de acuerdo al manual de Estándares Internacionales para la Medición Antropométrica (Stewart et al., 2011). Se realizaron por duplicado y verificando un error técnico de medición. Con la medida de cada valor se calcularon el IMC y el Índice de Cintura Cadera (ICC). También se determinó la masa magra (kg), y masa grasa en kilogramos $(\mathrm{kg})$ y el porcentaje de grasa como variable de composición corporal a partir de impedancia bioelectrica multifrecuencia (rango de frecuencia $5-200 \mathrm{kHz}$ ) con el equipo Quadscan 4000 (Bodystat, Islas Británicas).
El registro del nivel de AF realizada se documentó a través de la versión corta del cuestionario Internacional de Actividad Física (IPAQ) (Martinez et al., 2009; Craig et al., 2003) el cual fue aplicado mediante entrevista guiada. Con base en gasto reportado en equivalentes metabólicos (METs) por la práctica de $\mathrm{AF}$ se dividió a los sujetos en dos grupos de acuerdo a los METs gastados: AF alta $\geq 8 \mathrm{METs}$, AF moderadabaja $\geq 4$ METs.

Para determinar el $\mathrm{VO}_{2}$ máx se aplicó la prueba de campo Course Navette (Garcia et al., 2014) donde el sujeto debió correr de ida y vuelta una distancia de 20 metros previamente delimitados y señalados de forma visual; cuando el sujeto no completa una vuelta completa, entonces el investigador marca en una hoja de registro el nivel y la vuelta establecido como su máximo recorrido, al final de la prueba se toma en cuenta el número de vueltas y el nivel alcanzado, mismo que indicará su $\mathrm{VO}_{2}$ máx calculado mediante el predictor para consumo máximo de oxígeno (Ramsbottom, et al., 1988).

Dos días posteriores a la prueba de campo, se realizó una toma de muestra sanguínea venosa de los sujetos con un ayuno de 8 horas. Se utilizaron tubos con anticoagulante EDTA de $7.2 \mathrm{mg}$ marca BD Vacutainer, que se centrifugaron para obtener la fracción de plasma. Se conservaron alícuotas congeladas a $-20^{\circ} \mathrm{C}$ (Ultra-congelador marca Baxter Scientific Products Cryo-fridge) hasta su análisis. La determinación del SAT se llevó a cabo siguiendo la técnica de colorimetría bajo las especificaciones del kit para Capacidad Antioxidante Total Sigma (Sigma-Aldrich) con número de catálogo MAK187. Brevemente, se mezclaron $10 \mu \mathrm{L}$ de plasma (dilución 1:50 con agua destilada). Se incubaron durante $90 \mathrm{~min}$ a temperatura ambiente, tras los cuales se realizó la lectura utilizando un filtro para una absorbancia de $550 \mathrm{~nm}$ (espectrofotómetro BioTek ELx800). La concentración del SAT se ajustó en $\mu \mathrm{M}$ a partir de una curva estándar de Trolox, de acuerdo a la concentración de cada sujeto a $1 \mu \mathrm{g}$ de proteína plasmática.

Se realizó análisis exploratorio de los datos para verificar la calidad de los datos y en el caso de las variables medidas en escala de razón, se aplicó la prueba Kolmogorov-Smirnov para verificar que presentaban una distribución normal. Los datos se expresan como media y desviación estándar (D.E.) o mediana y rango intercuartil entre el 25 y 75 percentil. Las diferencias entre grupos $(p<0.05)$ se establecieron mediante la prueba t-Student para las variables de peso, talla, edad, porcentaje y total de grasa tanto en porcentaje como en kg, ICC, VO máx y SAT y la de suma de rangos de Wilcoxon. Para estudiar la relación entre $\mathrm{VO}_{2}$ máx y SAT se calculó el coeficiente de correlación de Pearson para las variables que no presentaron una distribución normal, mientras que para estudiar el nivel de AF y la relación con el SAT se utilizó la prueba de Wilcoxon. Se determinó un valor de $p<0.05$ como estadísticamente significativo. Los datos fueron analizados en paquete estadístico STATA versión 11.0 para Windows (College Station, TX: StataCorp).

\section{RESULTADOS}

El valor de las medias de las características generales, antropométricas y de composición corporal, se muestran en 
la Tabla 1, diferenciadas por género de los sujetos de estudio. Como se puede observar, el IMC es igual tanto en mujeres como en varones categorizando a los sujetos de estudio en peso adecuado, según la Organización Mundial de la Salud (OMS). En el caso de las variables de edad, peso, estatura, ICC y masa magra, los varones presentan valores mayores que las mujeres y sólo la masa grasa y porcentaje de grasa se encuentran en mayor proporción en ellas.

Tabla 1. Características antropométricas generales, composición corporal y edad.

Table 1. General anthropometric characteristics, body composition and age.

\begin{tabular}{|c|c|c|c|c|c|c|c|}
\hline & \multicolumn{3}{|c|}{ Mujeres $n=39$} & \multicolumn{4}{|c|}{ Varones $\mathrm{n}=17$} \\
\hline & Media & & D.E. & Media & & D.E. & $\mathbf{p}$ \\
\hline Edad (años) & 20.3 & \pm & 1.9 & 21.7 & \pm & 2.3 & 0.03 \\
\hline Peso (kg) & 61.6 & \pm & 8.9 & 69.4 & \pm & 8.8 & 0.004 \\
\hline Estatura (cm) & 160.9 & \pm & 6.1 & 171.8 & \pm & 7.9 & $<0.001$ \\
\hline IMC (kg/m²) & 23.8 & \pm & 3.0 & 23.5 & \pm & 2.3 & 0.66 \\
\hline ICC & 0.75 & \pm & 0.03 & 0.82 & \pm & 0.03 & $<0.001$ \\
\hline Grasa (\%) & 23.3 & \pm & 5.0 & 9.1 & \pm & 4.6 & $<0.001$ \\
\hline Grasa (kg) & 14.6 & \pm & 5.0 & 6.5 & \pm & 3.6 & $<0.001$ \\
\hline Masa magra $(\mathrm{kg})$ & 47.1 & \pm & 5.2 & 63.0 & \pm & 7.6 & $<0.001$ \\
\hline
\end{tabular}

$I M C=$ índice de masa corporal; $\mathrm{Kg}=$ Kilogramos; $\mathrm{cm}=$ centímetros; $I C C=$ Índice de Cintura-Cadera; D.S= Desviación estándar. ${ }^{*} p<0.05$

La medición de capacidad aerobia a través de la prueba de Course Navette, donde se muestran los datos de $\mathrm{VO}_{2}$ máx, la distancia recorrida durante la prueba diferenciada por grupo, el nivel del de actividad física reportado, así como las concentraciones de SAT en plasma se muestran en la Tabla 2.

En el grupo de varones se identificó un mayor valor de $\mathrm{VO}_{2}$ max y distancia recorrida que en mujeres. En ambos grupos en su mayoría alcanzaron un nivel máximo al realizar la prueba, frecuencia cardiaca máxima de 90 y $94 \%$ en los grupos de mujeres y varones respectivamente, al tomar de referencia los valores de $\mathrm{VO}_{2}$ max de Garber (Garber et al., 2011).

Al comparar los valores de SAT entre grupos se observó una tendencia $(p=0.08)$ a un valor mayor en mujeres que en los varones.

El análisis de correlación entre el SAT y las variables de estudio en ambos grupos se muestra que en varones se encontró una relación inversa del SAT $(p=0.019)$ con el valor de METs (Tabla 3).

\section{DISCUSIÓN}

En varones, el SAT estuvo inversamente relacionado con el número de METs reportados en el cuestionario de actividad física. Por otra parte, el SAT no se asoció con el VO2máx
Tabla 2. Datos de capacidad aerobia y SAT diferenciada por género.

Table 2. Aerobic capacity data and SAT differentiated by gender.

\begin{tabular}{|c|c|c|c|c|c|}
\hline \multirow[b]{2}{*}{$\mathrm{VO}_{2}$ máx (mL/kg/min) } & \multicolumn{2}{|c|}{$\begin{array}{c}\text { Mujeres n=39 } \\
\text { Media } \pm \text { D.E / n (\%) }\end{array}$} & \multicolumn{2}{|c|}{$\begin{array}{r}\text { Varones } \mathrm{n}=17 \\
\text { Media } \pm \text { D.E / } \mathrm{n}(\%)\end{array}$} & \multirow{2}{*}{$\frac{P}{0.01}$} \\
\hline & 34.5 & 7.6 & 43.6 & $\pm 7.0^{*}$ & \\
\hline Distancia recorrida (m) & 916.4 & $\pm \quad 443.0$ & 1471.8 & $\pm 453.8^{*}$ & 0.01 \\
\hline \multicolumn{6}{|l|}{ Nivel de actividad física } \\
\hline Leve/moderada & \multicolumn{2}{|r|}{$14(36)$} & \multicolumn{2}{|r|}{$6(35)$} & 0.96 \\
\hline Vigorosa & \multicolumn{2}{|r|}{$25(64)$} & \multicolumn{3}{|c|}{$11(65)$} \\
\hline SAT $\neq$ & \multicolumn{2}{|c|}{$0.84(0.77-0.94)$} & \multicolumn{2}{|c|}{$0.81(0.75-1.03)$} & 0.08 \\
\hline
\end{tabular}

$\mathrm{VO}_{2}$ máx= Consumo máximo de oxígeno; $\mathrm{mL}=$ mililitros; $\mathrm{kg}=\mathrm{kilogramo}$; min=minuto; $\mathrm{m}=$ metros; $\mathrm{SAT}=$ Sistema Antioxidante Total; $\mathrm{nm}=$ nano moles; $\mu \mathrm{L}=$ microlitro; $\mathrm{D} . \mathrm{E}=$ Desviación estándar. SAT ( $\mu \mathrm{M} / \mu \mathrm{\mu g}$ proteína) $\left({ }^{*} \mathrm{p}<0.05\right.$. $\neq=$ valores presentados en mediana y rango intercuartil entre el 25 y 75 percentil.

o variables de composición corporal. Los resultados sugieren una modulación del sistema antioxidante por la actividad física realizada, dependiente del género.

Componentes de la composición corporal como el IMC y el ICC, proponen parámetros en relación a la salud en población general (Cruz et al., 2009; Kweitel, 2007). Dentro de este estudio se observa que tanto los hombres como las mujeres se encuentran en su mayoría dentro del peso adecuado según la OMS (Barquera et al., 2013). Resultados similares se encontraron en diversas universidades de América Latina, en población de adultos jóvenes quienes presentaron un IMC dentro de este rango de normalidad (Saucedo-Molina et al., 2010; Yepez et al., 2008).

Tabla 3. Relación de las concentraciones de SAT con las variables de características generales y capacidad aerobia clasificada por género.

Table 3. Relationship of SAT concentrations with variables of general characteristics and aerobic capacity classified by gender.

\begin{tabular}{lcccc}
\hline VARIABLE & \multicolumn{2}{c}{ MUJERES } & \multicolumn{2}{c}{ VARONES } \\
& rho & P & r & p \\
\hline Edad (años) & 0.13 & 0.44 & 0.01 & 0.98 \\
\hline Peso $(\mathrm{kg})$ & -0.22 & 0.17 & -0.12 & 0.65 \\
\hline Estatura $(\mathrm{m})$ & -0.14 & 0.49 & -0.15 & 0.58 \\
Grasa $(\%)$ & -0.09 & 0.58 & 0.39 & 0.15 \\
Grasa $(\mathrm{Kg})$ & -0.16 & 0.34 & 0.37 & 0.18 \\
Magra $(\mathrm{Kg})$ & -0.15 & 0.35 & -0.32 & 0.26 \\
\hline IMC $(\mathrm{kg} / \mathrm{m} 2)$ & 0.04 & 0.82 & 0.30 & 0.92 \\
C. Cintura $(\mathrm{cm})$ & -0.17 & 0.29 & 0.07 & 0.79 \\
\hline C. Cadera $(\mathrm{cm})$ & -0.21 & 0.21 & -0.06 & 0.84 \\
ICC & $<.01$ & 1.0 & 0.35 & 0.18 \\
\hline METs & 0.02 & 0.91 & -0.58 & 0.02 \\
VO ${ }_{2}$ máx $(\mathrm{ml} / \mathrm{kg} / \mathrm{min})$ & 0.03 & 0.85 & -0.35 & 0.18 \\
\hline
\end{tabular}

* $\mathrm{p}<0.05, \mathrm{IMC}=$ Índice de masa corporal; Kg / m2= Kilogramo por metro cuadrado; $\mathrm{Kg}=$ Kilogramos; $\mathrm{cm}=$ centímetros; ICC=Índice cintura-cadera. ₹Nivel de actividad física alta vs. Ligera-moderada. $\mathrm{Rho}=$ coeficiente de correlación de Spearman, r=coeficiente correlación de Pearson. 
La distribución de tejido adiposo y tejido magro ha sido apoyada por la literatura en diversas ocasiones, como parte de estos parámetros de salud se menciona que generalmente las mujeres tienen una mayor adiposidad en comparación a los hombres durante el transcurso de la vida, coincidiendo con estudios como el de Ochoa-Martinez et al. (2018) quien tras evaluar una muestra de estudiantes universitarios con una edad promedio similar a la de nuestro estudio (20.67 \pm 1.69 años) a través de impedancia bioeléctrica encuentran un mayor porcentaje de grasa en las mujeres que en los hombres, sin embargo en este trabajo dicho valor fue menor en ambos sexos. Los resultados de otras investigación muestran que el ICC es mayor en hombres que en mujeres, lo que puede atribuirse a un almacenamiento o distribución del tejido que es seccionalmente diferente por género (White et al., 2014; Gallagher et al., 1996). Los datos anteriormente mencionados presentan similitud en este estudio, ya que existe un aumento en los valores de las variables de grasa corporal (porcentaje y kilogramos), siendo mayor para el caso de las mujeres y mayor cantidad de masa magra en los varones.

Esta investigación aporta información sobre los valores de $\mathrm{VO}_{2}$ máx en varones, que son similares a los obtenidos en una investigación realizada en el 2015 por Hall-López et al., en estudiantes físicamente activos con una edad promedio de $23.4 \pm 1.3$ y un $\mathrm{VO}_{2}$ máx $43.6 \pm 7.0$, medidos por prueba de campo como Course Navette y en prueba de esfuerzo en ergómetro con el protocolo de Bruce; a pesar de ser pruebas diferentes se observa similitud en los valores reportados.

La relación entre el sistema antioxidante y el ejercicio ha sido reportada por varios autores. Ramos en el 2014 estudió a 160 adultos con una edad entre 35 y 60 años, los cuales no realizaban actividad física previa al estudio, la intervención consistió en un programa de ejercicio aerobio con una intensidad del $60 \%$ del $\mathrm{VO}_{2}$ máx. Sus resultados indicaron un aumento en el sistema antioxidante, utilizando como indicador las concentraciones de la proteína antioxidante catalasa en plasma (Ramos, 2014). A pesar de que a los participantes de este estudio no se les aplicó intervención, presentan menor edad, no se encontramos similitudes en el grupo de mujeres en cuanto a la relación de antioxidantes, con ello del $\mathrm{VO}_{2}$ máx y el SAT.

Otro estudio menciona que las mujeres responden adecuadamente en relación a la actividad física y la expresión génica de enzimas antioxidantes, ya que se encontró una mayor concentración de la enzima superóxido dismutasa (Mn-SOD) 3 horas post ejercicio $(P<0,05)$ (Baghaiee et al., 2016). Si bien en nuestros grupos no se evaluó dicha enzima, de forma general como sistema antioxidante no se observaron diferencias por género en dicha variable.

Algunos autores asocian el estrés oxidativo con el entrenamiento aerobio, tanto en humanos como en modelos animales, quienes concuerdan que el entrenamiento incrementa los niveles de lipoperoxidación mientras que el sistema antioxidante se ve disminuido o modulado de acuerdo a la intensidad (Poblete et al., 2015; Matheus et al., 2016;
Requena, 2012). Como Matheus et al. (2016), quienes reportaron una protección por efecto del ejercicio en un modelo animal, a través del incremento de SAT y una disminución de un marcador de daño oxidativo. Si bien los datos obtenidos en este estudio asocian una disminución del SAT a mayor AF realizada ( $>$ METs), sin la aplicación de ejercicio al momento de su medición, lo que nos lleva a sugerir que quizá el sistema antioxidante no es tan eficiente en la eliminación de su contraparte cuando se realiza AF vigorosa.

En la literatura consultada, poca se reporta la modulación del SAT por efecto de la aplicación de ejercicio (Baghaiee et al., 2016; Majerczak et al., 2010; Gawron-Skarbek et al., 2015), sin embargo, el único reporte que presenta evidencia directa entre las variables del presente estudio, es Rahimi et al. (2014) quienes establecen una relación directa del $\mathrm{VO}_{2}$ máx y SAT en varones adultos con asma y quienes además presentan condiciones de sobrepeso y obesidad. En este trabajo es notable observar que en el grupo de adultos jóvenes sanos se encuentra ausente la relación entre dichas variables.

\section{CONCLUSIONES}

Se concluye que existe una relación indirecta entre el SAT y la AF reportada sólo en los varones. Sin embargo, se requiere de una caracterización específica del contenido antioxidante y su contraparte como radicales libres para tener un panorama más completo y lograr información que nos permita monitorear al momento de aplicar ejercicio en adultos jóvenes.

\section{AGRADECIMIENTOS}

LAEC es un receptor de beca adscrito al Consejo Nacional de Ciencia y Tecnología (CONACyT), México D.F.

\section{REFERENCIAS}

Abarca-Sos, A., Casterad, Z., Lanaspa, G. y Clemente, J. 2010. Comportamientos sedentarios y patrones de actividad física en adolescentes. Revista Internacional de Medicina y Ciencias de la Actividad Física y Deporte. 10 (39): 410-427.

Aguiló, A., Tauler, P., Sureda, A., Cases, N., Turb, J. y Pons, A. 2007. Antioxidant diet supplementation enhances aerobic performance in amateur sportsmen. Journal of Sports Sciences. 25(11): 1203-1210.

Ainsworth, E. A. 2008. Rice production in a changing climate: a meta-analysis of responses to elevated carbon dioxide and elevated ozone concentration. Global Change Biology. 14(7): 1642-1650.

Asociación Médica Mundial. 2014. Declaración de Helsinki. Principios éticos para la investigación en seres humanos. Boletín del Consejo Académico de Ética en Medicina. 1(2): 239-243.

Baghaiee, B., Aliparasti, M. R., Almasi, S., Siahkuhian, M. y Baradaran, B. 2016. Antioxidant Expression Response to Free Radicals in Active Men and Women Fallowing to a Session Incremental Exercise; Numerical Relationship Between Antioxidants and Free Radicals. Asian Journal of Sports Medicine. 7 (2): 1-9. 
Barquera, S., Campos-Nonato, I., Hernández-Barrera, L., Pedroza, A. y Rivera-Dommarco, J. A. 2013. Prevalence of obesity in Mexican adults 2000-2012. Salud Pública de México 55: 151160.

Craig, C.L., Marshall, A.L., Sjostrom, M., Bauman, A.E., Booth, M.L., Ainsworth, B.E. 2003. International physical activity questionnaire: 12-country reliability and validity. Medicine and Science in Sports and Exercise, 35: 1381-95.

Cruz, J. R. A., Armesilla, M. D. C. y de Lucas, A. H. 2009. Protocolo de valoración de la composición corporal para el reconocimiento médico-deportivo. Documento de consenso del grupo español de cineantropometría de la federación española de medicina del deporte. Archivos de medicina del deporte: Revista de la Federación Española de Medicina del Deporte y de la Confederación Iberoamericana de Medicina del Deporte. 131: 166-179.

Gallagher, D., Visser, M., Sepulveda, D.,Pierson, R. N.,Harris, T. y Heymsfield, S. B. 1996. How useful is body mass index for comparison of body fatness across age, sex, y ethnic groups? . American Journal of Epidemiology. 143 (3): 228-239.

Garber, C. E., Blissmer, B., Deschenes, M. R., Franklin, B. A., Lamonte, M. J., Leelarungrayub, I.-M., Neiman, D.C. y Swain, D. P. 2011. American College of Sports Medicine position stand. Quantity and quality of exercise for developing and maintaining cardiorespiratory, musculoskeletal, and neuromotor fitness in apparently healthy adults: guidance for prescribing exercise. Medicine and Science in Sports and Exercise. 43(7): 1334-1359.

Garcia Gastón, C. y Secchi Jeremías, D. 2014. Test course navette de 20 metros con etapas de un minuto. Una idea original que perdura hace 30 años. Apunts Medicina de L'sport. 49 (183): 93-103.

Gawron-Skarbek A., Chrzczanowicz J., Kostla J., Nowak D., Drygas W., Jegier A. y Kostka T. 2015. Physical activity, aerobic capacity, and total antioxidant capacity in healthy men and in men with coronary heart disease. Oxidative Medicine and Cellular longevity. (2015): 1-9.

Gutierrez Salinas, J. 2007. Función de los complementos antioxidantes durante el ejercicio. Medicina Interna de México. 23(3): 218-222.

Hall-López, J. A., Ochoa-Martínez, P. Y., Moncada-Jiménez, J., Méndez, M. A., García, I. M., y García, M. A. (2015). Reliability of the maximal oxygen uptake following two consecutive trials by indirect calorimetry. Nutrición Hospitalaria. 31 (4): 1726-1732.

Karvonen, M. J., Kentala, E. y Mustala, O. 1957. The effects of training on heart rate: A longitudinal study. Annales Medicinae Experimentalis et Biologiae Feenniae. 35(3): 30715.

Konigsberg Fainstein, M. 2007. Nrf2: La historia de un nuevo factor de transcripción que responde a estrés oxidativo. REB. 18(25): 18-23.

Kweitel, S. 2007. IMC: Herramienta poco útil para determinar el peso ideal de un deportista. Rev. Internacional de Medicina y Ciencias de la Actividad Física y el Deporte. 7(28): 274-289.

Majerczak, J., Rychlik, B., Grzelak, A., Grzmil, P., Karasinski, J. y Pierzchalski, P. Zoladz, J. 2010. Effect of 5-week moderate intensity endurance training on the oxidative stress, muscle specific uncoupling protein (UPC3) and superoxide dismutase (SOD2) contents in vastus lateralis of young healthy men. Journal of Physiology and Pharmacology. 61(6): 743-751.
Martinez, D., Martinez-De-Haro V., Del Campo J., Zapatera B., Welk G., Villagra A., Marcos A. y Veiga O. 2009. Validez de cuatro cuestionarios para valorar la Actividad Física en Estudiantes Españoles. Gaceta Sanitaria. 23 (6): 512-517.

Martinez-Vizcaino, V. y Sanchez-Lopez, M. 2008. Relación entre la AF física y condición física en niños y adolescentes. Revista Española de Cardiología. 61: 108-111.

Matheus, N. J., Mendoza, C. A., Meléndez, C., Flores, C. A., Corro, A. C., Medina, I. C. y Báez, E. 2016. Entrenamiento Aeróbico: Efecto Sobre el Estado Oxidativo Hepático. Revista Internacional de Ciencias del Deporte. 12 (45): 309-323.

Ochoa-Martinez, P., Hall-López, J., Solano-Pineda, I., Monreal Ortiz, L., Chacón-Araya, Y., y Moncada-Jiménez, J. 2017. Prediction of body fat through body adiposity index and bioelectrical impedance analysis in a sample of physically active Mexican students. Retos. 0 (34), 128-131.

Ozcelik, O., Ozkan, Y., Karatas, F. y Kelestimur, H. 2005. Exercise training as an adjunct to orlistat therapy reduces oxidative stress in obese subjects. The Tohoku Journal of Experimental Medicine. 206 (4): 313-318.

Peake, J. y Suzuki, K. 2004. Neutrophil activation, antioxidant supplements and exercise-induced oxidative stress. Exercise Immunology Review. 10(1): 129-141.

Poblete Aro, C. E., Russell Guzmán, J. A., Soto Muñoz, M. E. y Villegas Gonzáles, B. E. 2015. Efectividad del entrenamiento interválico de alta intensidad comparado con entrenamiento continúo de moderada intensidad en la reducción de estrés oxidativo de pacientes adultos con diabetes mellitus tipo 2: CAT. MEDwave. 15(7): 239-248.

Quintanar Escorza, M. A. y Calderón Salinas, J. V. 2009. La capacidad antioxidante total. Bases y aplicaciones. Revista de Educación Bioquímica, 28(3): 89-101.

Rahimi, A., Kasbparast Jr, M. y Shokrgozar, A. 2014. Aerobic capacity and Antioxidant profile in asthma patients. Biological Forum. 6 (2): 509-513.

Ramos Mejías, A. S. 2014. Estudio de estrés oxidativo en pacientes que siguen o no un programa de rehabilitación cardíaca: Disertación doctoral. Universidad de Granada.

Ramsbottom, R., Brewer, J. y Williams, C. 1988. A progressive shuttle run test to estimate maximal oxygen uptake. British Journal of Sports Medicine. 22(4): 141-144.

Requena Domenech, F. 2012. Evaluación de la capacidad física del toro de lidia con el entrenamiento. Tesis doctoral. Universidad de aCórdoba.

Sacheck, J. M. y Blumberg, J. B. 2001. Role of vitamin E and oxidative stress in exercise. Nutrition. 17(10): 809-814.

Saucedo-Molina, T. D J. y Unikel Santoncini, C. 2010. Conductas alimentarias de riesgo, interiorización del ideal estético de delgadez e índice de masa corporal en estudiantes hidalguenses de preparatoria y licenciatura de una institución privada. Salud Mental. 33(1): 11-19.

Serra Puyal, J. R. 2008. Factores que influencian la práctica de la Actividad física en la población adolescente de la provincia de Huesca, Tesis doctoral. Universidad de Zaragoza.

Stewart, A., Marfell-Jones, M., Olds, T.y Hans, d. R. 2011. Protocolo internacional para la valoración antropométrica (1 1 $^{\mathrm{a}}$ ed.). United Kingdom. Sociedad Internacional para el Avance de la Cineantropometría.

Tobias, E., Perikles, S. y Dirk A., M. 2013. Epigenetic in sports. Sports Medicine. 43(2): 93-110. 
Torres Rios, A., Fuentes, L., Castro, A. y Aguilar Carbo, F. 2015. Antioxidantes como alimentos funcionales. Tesis. Universidad Autónoma Agraria Antonio Narro. Saltillo, Coahuila.

Valdés Casique, L. M. 2014. Tasa de recuperación de la frecuencia cardiaca al minuto de post esfuerzo máximo, en un equipo de segunda división profesional de futbol asociación, como indicador de mortalidad. Toluca México 2013. Tesis Doctoral. Universidad Autónoma del Estado de México.
Velamazán, A. 2005. Antioxidantes: una respuesta natural. Medicina Naturista. 8: 421-428.

White, U. A. y Tchoukalova. D. 2014. Sex dimorphism and depot differences in adipose tissue function. Biochimica et Biophyphica Acta (BBA). Molecular Basis of Disease, 1842 (3): 377-392.

Yepez, R., Carrasco, F. y Baldeón, M. E. 2008. Prevalencia de sobrepeso y obesidad en estudiantes adolescentes ecuatorianos del área urbana. Archivos Latinoamericanos de Nutrición. 58(2): 139-143. 